\title{
Simple technique for replication of micro-optical elements
}

\author{
Philippe Nussbaum \\ Irène Philipoussis \\ Aline Husser \\ Hans Peter Herzig \\ University of Neuchâtel \\ Institute of Microtechnology \\ Rue A. L. Breguet 2, \\ $\mathrm{CH}-2000$ Neuchâtel \\ Switzerland \\ E-mail: phillippe.nussbaum@imt.unine.ch
}

\begin{abstract}
A simple technique for the replication of micro-optical elements is presented. Elastomeric material is used to realize a negative mold of the original optical element and UV-curing adhesive is used to make the replicated copy. Replicated elements, such as refractive and diffractive micro-optical elements, are produced. The refractive microlenses have a diameter of $970 \mu \mathrm{m}$ and a height of $79 \mu \mathrm{m}$ and the diffractive element is a multilevel blazed grating with a period of $64 \mu \mathrm{m}$. The characteristics of the replicated elements are measured using different methods. The deviation from a sphere of the original and the replicated refractive microlenses is $0.13 \lambda$ and $0.12 \lambda$ root mean square ( $\mathrm{rms}$ ), respectively. The replicated multilevel blazed grating has a diffraction efficiency of over $80 \%$. An alternative method for realizing planoconcave microlenses from a planoconvex master is also presented. () 1998 Society of Photo-Optical Instrumentation Engineers. [S0091-3286(98)01106-4]
\end{abstract}

Subject terms: replication; micro-optical elements; microsystems; elastomeric material.

Paper 37097 received Sep. 29, 1997; revised manuscript received Jan. 13, 1998; accepted for publication Jan. 14, 1998.

\section{Introduction}

The replication of surface-relief micro-optical elements, such as refractive microlens arrays or diffractive optical elements (DOEs), is of great interest for optical microsystems. ${ }^{1}$ Compared to the initial photoresist element, the replicated element offers high transmission and reduced material fluorescence. Standard replication techniques, such as hot embossing, molding, or casting, are key technologies for low-cost mass-production of micro-optical elements. ${ }^{2}$ In contrast to these well-known techniques, we investigate a new method that offers a facility to replicate optical elements without requiring complicated and expensive infrastructures, such as sputtering chambers, electroplating baths, embossing machines, or injection molding systems. This method was first applied to optical elements by Wilbur et $\mathrm{al}^{3}{ }^{3}$ and Xia et al. ${ }^{4}$ Their papers present the basic principle and some mechanical properties of the elements. In a more recent publication, Daly et al. ${ }^{5}$ used the same technique to replicate refractive microlens arrays with elastomeric material for the mold. They used thermally curable epoxy for the replicated element.

We report on the optical properties of replicated microoptical elements. For the replication we use the same commercially available elastomeric material to realize the mold as in Ref. 5, but we use a UV-curing adhesive for the replication. The elements were refractive microlens arrays, having features up to $1.4 \mathrm{~mm}$ diameter and $95 \mu \mathrm{m}$ lens height, and multilevel diffractive blazed gratings, having up to eight levels and periods down to $2 \mu \mathrm{m}$. Different characterization methods, such as accurate interferometric analysis of the microlens shape or diffraction efficiencies of the blazed gratings, are used to analyze the performance of the replications. An alternative method to realize good quality planoconcave refractive microlenses is also pre- sented. First, we describe the simple replication method and then we present results for the realized elements.

\section{Fabrication Method}

The fabrication of replicated elements is realized in two steps. The first step consists of making the negative mold of the original element, and the second step is the replication in UV-curing adhesive, as shown in Fig. 1. The original micro-optical element is first prepared by a cleaning procedure using a nitrogen jet, if the original element is made of photoresist, or using solvents, if it is made of a hard material such as glass or silicon. The elastomeric material used for the mold is commercially available from Dow Corning (Sylgard 184). This material is a two-component silicon elastomer developed for encapsulating and protecting electronic circuits or solar cells against external influences such as shocks or dust particles. For the preparation of the moulding material, the manufacturer recommends a mixture of 1 part of catalyzer for 10 parts of elastomer. We also tried other mixtures with less success. After pouring the mixture onto the original master, both are put in an oven and baked at $50^{\circ} \mathrm{C}$ for one night $(12 \mathrm{~h})$. Once the elastomer is hardened, it is very simple to separate the mold from the original master. The adhesion of both parts is only by vacuum, thus the original master remains intact after the separation. This property can be an important issue if several molds must be realized from the same original master.

For the replication step, a standard UV-curing adhesive is used. The NOA 61 from Norland Optical Adhesives shows very good results for refractive and diffractive micro-optical elements. The adhesive presents good optical quality, a refractive index of 1.56 , and good transmission for visible and IR light. For the UV region below $400 \mathrm{~nm}$, the adhesive has a high absorbance, as do all organic ma- 


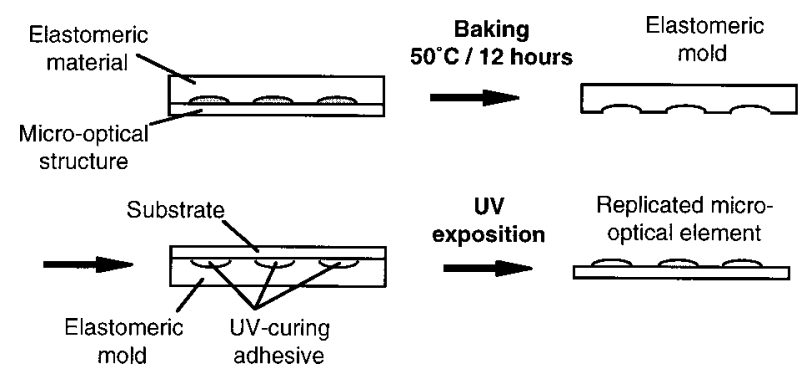

Fig. 1 Replication of micro-optical elements using elastomeric molds and UV-curing adhesive.

terials. A small droplet of this NOA 61 adhesive is poured on the elastomeric mold. Care must be taken to avoid air bubbles. A substrate (e.g., glass, quartz, or plastic) is put on top of this adhesive layer to provide a flat optical substrate for the replicated element. For the polymerization, a 500-W flood exposure mercury lamp from Oriel is used. It is important that the adhesive is well cured under UV light. In our case, an exposure of $20 \mathrm{~min}$ with an intensity of 10 $\mathrm{W} / \mathrm{cm}^{2}$ was found to be ideal. Finally, the separation of the replicated micro-optical element from the elastomeric mold is again very simple.

\section{Experimental Results}

\subsection{Refractive Microlenses}

Refractive microlens arrays were used as original masters to verify the quality of this replication method. The original microlens array was realized using the melting resist technology, where photoresist pedestals were obtained by photolithographic steps. ${ }^{6,7}$ A melting step forms planoconvex microlenses. Using this technique, the realized elements were quasi-perfect parts of a sphere. ${ }^{8}$ The replication technique described in the preceding section was used to make copies of these microlenses. The diameter of the lenses was
$970 \mu \mathrm{m}$ and the height was $79 \mu \mathrm{m}$. A Twyman-Green interferometer was used to characterize the replicated elements. ${ }^{9}$ The results were compared to the measurements of the original master. Figure 2 shows the deviation from sphere of the original resist microlens and the deviation from sphere of the microlens replicated in UV-curing adhesive. The observed deviation from sphere was $0.13 \lambda$ root mean square $(\mathrm{rms})$ and $1.11 \lambda$ peak-to-valley $(p / v)$ for the original master and $0.12 \lambda$ (rms) and $1.00 \lambda$ peak-to-valley $(p / v)$ for the replicated microlens.

An atomic force microscope (AFM) was used to measure the surface roughness of the original photoresist master microlens and of the replicated epoxy microlens. A surface roughness of 2 to $3 \mathrm{~nm}$ (rms) was measured for the original photoresist element and about $4 \mathrm{~nm}(\mathrm{rms})$ for the replicated structure. These surface roughness values are negligible for visible light and are of less importance for UV light since the UV-curing adhesive has low transmission in this wavelength domain $(30 \%$ transmission at 300 $\mathrm{nm}$ for NOA 61 adhesive).

Replication of micro-optical elements provides high fidelity of the geometrical shape and dimension: diameter, lens height, or radius of curvature of the original element. Due to the change of material between the original and the replicated element, the optical properties change also. A change in the refractive index, for example, directly affects the focal length of the replicated element. This fact must be taken into account in the design of a replicated microoptical element.

\subsection{Planoconcave Replicated Microlens from a Planoconvex Original Master}

The melting resist technology is limited to the fabrication of planoconvex lenses. Therefore it is interesting to realize plano-concave lenses by replication. The mold of a 1.4-mm-diam and 95- $\mu \mathrm{m}$-height microlens was realized. This planoconcave mold served as a master for a second (a)

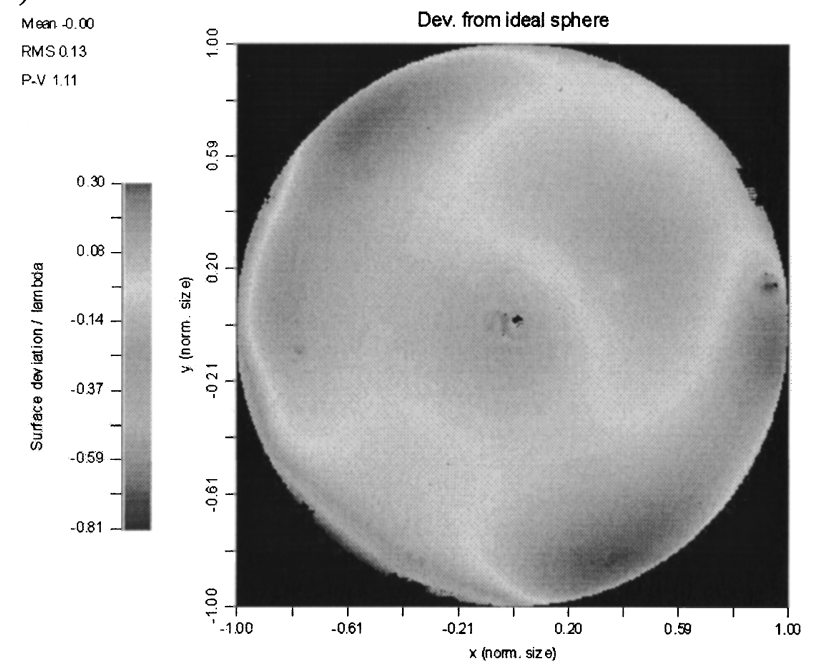

(b)

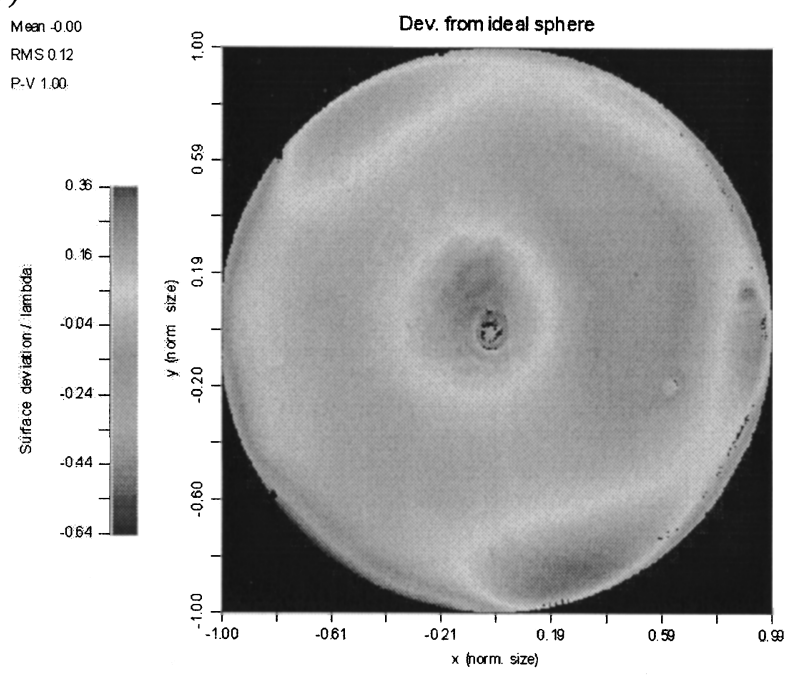

Fig. 2 Deviation from sphere measured with a Twyman-Green interferometer: (a) original resist master and (b) replicated element. The diameter of the microlens is $970 \mu \mathrm{m}$ and the height is $79 \mu \mathrm{m}$. 
(a)

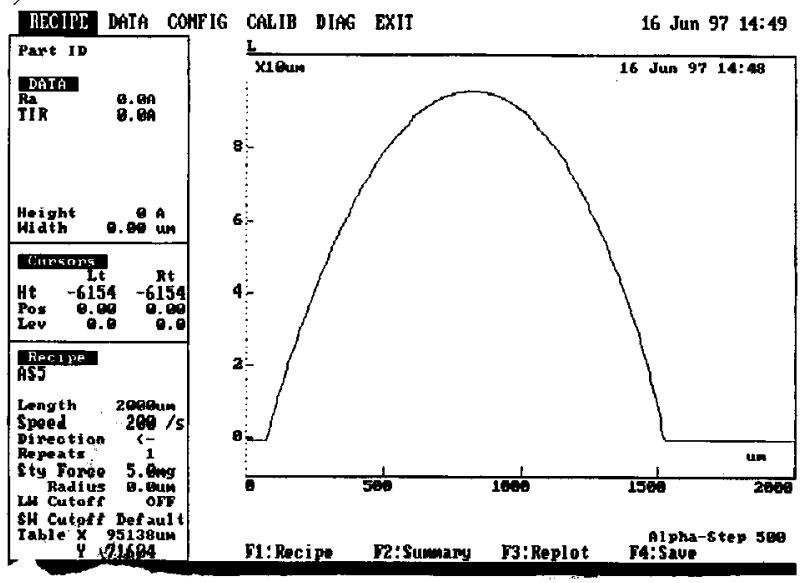

(b)

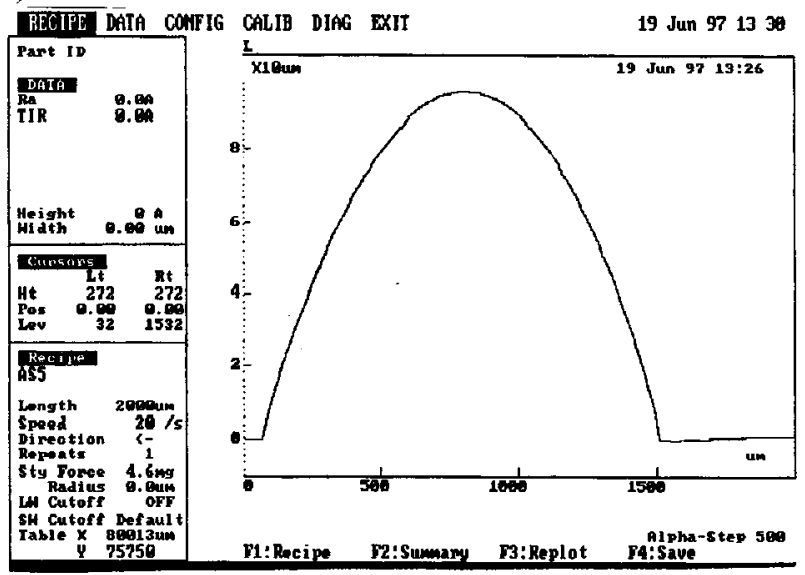

(c)

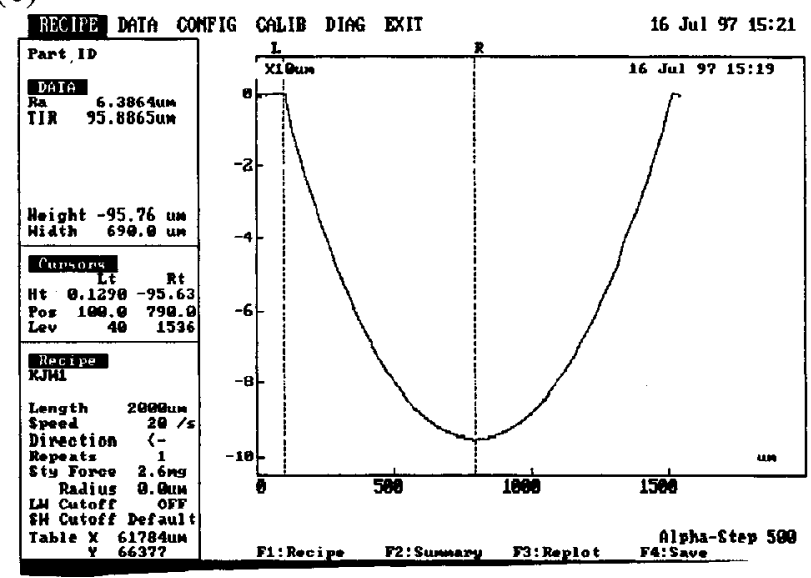

Fig. 3 Surface profiles measured with a stylus profilometer: (a) original planoconvex resist microlens master, (b) replicated planoconvex microlens, and (c) replicated planoconcave microlens. The diameter of the lens is $1.4 \mathrm{~mm}$ and the height is $95 \mu \mathrm{m}$.

molding. From this second planoconvex elastomeric mold, a replication was made using UV-curing adhesive as described previously. The achieved element was a replicated planoconcave microlens. Figure 3 shows the surface profile, measured with a Tencor stylus profilometer, of the original planoconvex resist microlens master, the replicated planoconvex microlens, and the replicated planoconcave microlens.

The replicated planoconvex and planoconcave elements have the same geometrical dimensions. The measured surface profiles show the same height of $95 \mu \mathrm{m}$ for a diameter of $1.4 \mathrm{~mm}$ for the three elements (Fig. 3). No significant error is observed between the profiles. A Twyman-Green interferometer is well suited to measure the deviation from sphere of a planoconvex microlens. However, it is more difficult to get an accurate result for planoconcave spherical profiles. In this work, it was not possible to measure the deviation from sphere of the planoconcave replicated element. In the future, modifications in the setup and in the interferometer software must be realized to adapt the method also to planoconcave spherical shapes.

\subsection{Multilevel Blazed Gratings}

Multilevel blazed gratings served as masters for replication of diffractive micro-optical elements. The original DOE was realized in different sequences using a series of lithographic and etching steps. ${ }^{10}$ The obtained structure is a socalled multilevel blazed grating. Replication using elastomeric material and UV-curing adhesives was performed on this type of element. We replicated multilevel blazed gratings with periods from 64 to $8 \mu \mathrm{m}$ having eight phase levels with good results. Figure 4 shows scanning electron microscopy (SEM) pictures of an original master and a replicated multilevel blazed grating $(64 \mu \mathrm{m}$ period blazed grating having eight levels).

The diffraction efficiencies of the different elements were analyzed. The diffraction efficiency is defined as the ratio between the power in the first diffraction order and the power of the incident beam. The elements were illuminated with a He-Ne laser and the intensity in the first diffraction order was measured. For the original blazed grating (64$\mu \mathrm{m}$ period blazed grating having eight levels), an efficiency of $84.7 \%$ was obtained. The efficiency of the replicated 
(a)

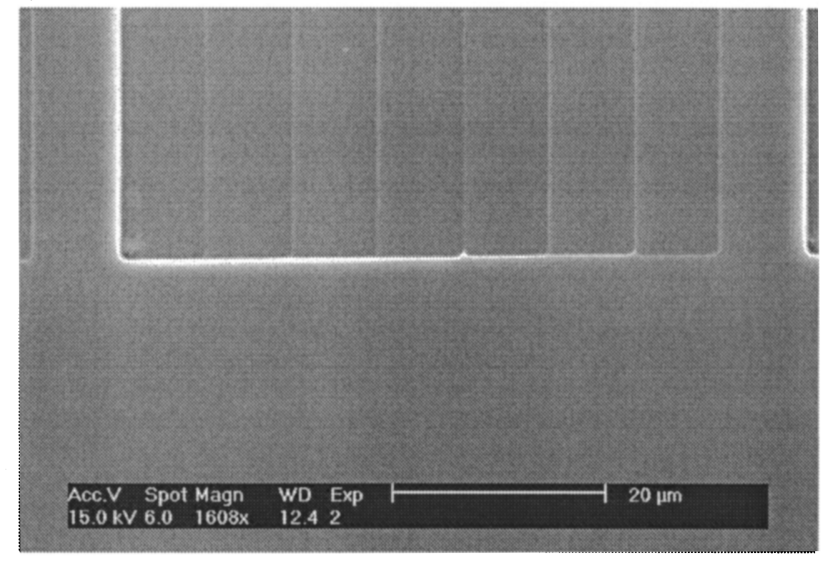

(b)

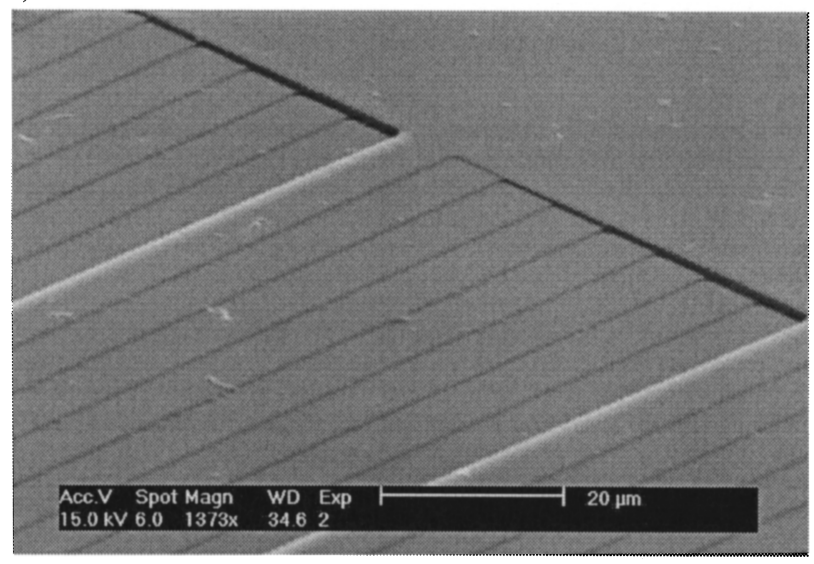

Fig. 4 SEM pictures of (a) the original master and (b) the replicated element. The period of the eight-level blazed grating is $64 \mu \mathrm{m}$.

multilevel blazed grating at the same wavelength was $77.9 \%$. The important difference in the diffraction efficiencies of the original and the replicated elements is due to a different phase delay for the two elements. In this case, the original quartz element was designed for $\mathrm{He}-\mathrm{Ne}$ wavelength of $633 \mathrm{~nm}$ with a refractive index of 1.457. The replicated element has the same grating depth but another phase delay because of the refractive index of 1.557 at 633 $\mathrm{nm}$. The appropriate wavelength for the phase delay of the replicated element is around $790 \mathrm{~nm}$. A tunable Ti:Sapphire laser at a wavelength of $790 \mathrm{~nm}$ was used to measure the diffraction efficiency. An efficiency in the first diffraction order of $84.8 \%$ was obtained.

\section{Conclusions}

We demonstrated the fabrication of good-quality replicated micro-optical elements using elastomeric molds. This simple technique is very promising for small replication series. Good results for both refractive microlens arrays and DOEs were obtained. No significant difference between the original masters and the replicated elements was noticed. The fact that the original master is still intact after the process is an advantage if several molds must be realized from the same original element. We also demonstrated that this technique is suitable for the realization of inverted copies. Planoconcave microlenses were produced from a planoconvex original master microlens.

Standard micro-optical elements are always realized on a hard carrier substrate having a thickness of several hundred micrometers. In some cases, such as the integration of micro-optical elements in microsystems, these bulky substrates are not acceptable. Using elastomeric molds, it is possible to use them as stamps to create isolated microoptical elements, which can be integrated directly in microsystems without bulky carrier substrates.

\section{Acknowlegments}

The work was supported by the Swiss Priority Program OPTIQUE. The authors like to thank R. Voelkel, C. Ossman, A. Schilling of IMT-Uni Neuchâtel, and K. J. Weible of Weible Optech for their help.

\section{References}

1. H. P. Herzig, Ed., Micro-Optics: Elements, Systems, and Applications, Taylor \& Francis, London (1997)

2. M. T. Gale, "Replication,"' in Micro-Optics: Elements, Systems, and Applications, H. P. Herzig, Ed., pp. 87-126, Taylor \& Francis, London (1997)

3. J. L. Wilbur, R. J. Jackman, G. M. Whitesides, E. L. Cheung, L. K. Lee, and M. G. Prentiss, "Elastomeric optics," Chem. Mater. 8, 1380-1385 (1996)

4. Y. Xia, E. Kim, X.-M. Zhao, J. A. Rogers, M. Prentiss, and G. M. Whitesides, "Complex optical surfaces formed by replica molding against elastomeric masters," Science 273, 347-349 (1996).

5. D. J. Daly, R. A. Ferguson, and M. C. Hutley, "'Replication of optical components using silicone rubber intermediate replica," Proc. SPIE 3099, 83-88 (1997).

6. Z. D. Popovic, R. A. Sprague, and G. A. Neville-Connell, "Technique for the monolithic fabrication of microlens arrays,', Appl. Opt. 27, 1281-1284 (1988).

7. D. Daly, R. F. Stevens, M. C. Hutley, and N. Davies, "The manufacture of microlenses by melting photoresist,', J. Meas. Sci. Technol. 1, 759-766 (1990).

8. P. Nussbaum, R. Voelkel, H. P. Herzig, M. Eisner, and S. Haselbeck, "Design, fabrication and testing of microlens arrays for sensors and microsystems,', Pure Appl. Opt. 6, 1-20 (1997).

9. J. Schwider and O. Falkenstörfer, "Twyman-Green interferometer for testing microspheres,' Opt. Eng. 34, 2972-2975 (1995).

10. M. B. Stern, "Binary optics fabrication,', in Micro-Optics: Elements Systems, and Applications, H. P. Herzig, Ed., pp. 53-86, Taylor \& Francis, London (1997).

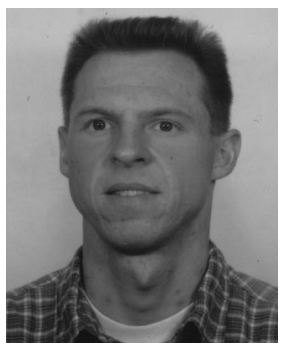

Philippe Nussbaum studied optics in France, where he received his diploma in 1990. In 1992 he was in the Analytical Research Department of the Ciba-Geigy Company in Basel, Switzerland, working on the development of miniaturized optoelectronic sensors for chemical analysis. Since 1993, he has been with the Applied Optics Group at the Institute of Microtechnology of the University of Neuchâtel, Switzerland. He is in charge of the refractive and diffractive micro-optical technology. Philippe Nussbaum is member of the Swiss Society for Optics and Microscopy. 


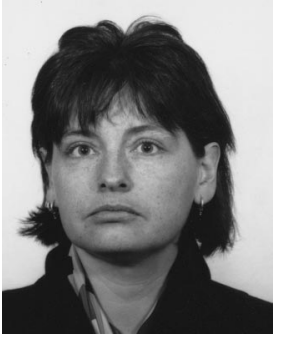

Irène Philipoussis did her apprenticeship as a physics technician at the European Organization for Nuclear Research (CERN) laboratories in Geneva, Switzerland. From 1986 to 1990 she was with Oscilloquartz in Neuchâtel, Switzerland, working on atomic frequency standards. From 1990 to 1996, she was with the Liquid Crystal Display Group of the Asulab SMH Central Laboratories in Neuchâtel, Switzerland. In 1997, she joined the Applied Optics Group at the Institute of Microtechnology of the University of Neuchâtel, Switzerland. She is in charge of the fabrication of micro-optical elements.

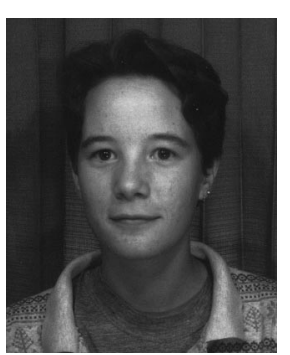

Aline Husser is a student at the Lycée d'Enseignment Général et Technique (LEGT) in Saint-Louis, France, where she is preparing her diploma in optics. In 1997 she was with the Applied Optics Group at the Institute of Microtechnology of the University of Neuchâtel, Switzerland, studying the performance of elastomeric materials for replication techniques.

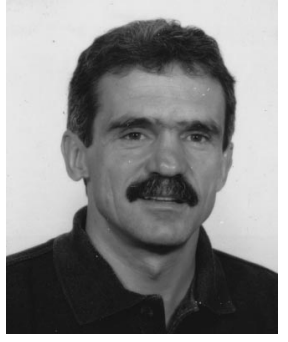

Hans Peter Herzig received the diploma in physics from the Swiss Federal Institute of Technology in Zürich, Switzerland, in 1978. From 1978 to 1982 he was a scientist with the Optics Development Department of the Kern Company in Aarau, Switzerland, working on lens design and optical testing. In 1983, he joined the Applied Optics Group at the Institute of Microtechnology of the University of Neuchâtel, Switzerland, as a graduate research assistant, working in the field of holographic optical elements, especially scanning elements. In 1987, he received his PhD degree in optics. He currently heads the Micro-Optics Research Group and Privat-docent at the University of Neuchâtel. Dr. Herzig is member of OSA and EOS and a board member of the Swiss Society for Optics and Microscopy. 